\title{
ENERGY EFFICIENT GRID AND TREE BASED ROUTING PROTOCOL
}

\author{
Reshma $\mathbf{J}^{1}$, Kavitha $\mathrm{C}^{2}$ and Malashri $\mathrm{S}^{3}$ \\ ${ }^{1}$ Research Scholar, Department of Computer Science \& Engineering, \\ B N M Institute of Technology, Bengaluru \\ ${ }^{2}$ Professor \& Head, Department of Information Science \& Engineering, \\ B N M Institute of Technology, Bengaluru \\ ${ }^{3} \mathrm{M}$. Tech Student, B N M Institute of Technology, Bengaluru
}

\begin{abstract}
In Wireless Sensor Network, a large number of sensor nodes are deployed and they mainly consume energy in transmitting data over long distances. Sensor nodes are battery powered and their energy is restricted. Since the location of the sink is remote, considerable energy would be consumed if each node directly transmits data to the base station. Aggregating data at the intermediate nodes and transmitting using multihops aids in reducing energy consumption to a great extent. This paper proposes a hybrid protocol "Energy efficient Grid and Tree based routing protocol" (EGT) in which the sensing area is divided into grids. The nodes in the grid relay data to the cell leader which aggregates the data and transmits to the sink using the constructed hop tree. Simulation results show that EGT performs better than LEACH.
\end{abstract}

\section{KEYWORDS}

Wireless Sensor Network, Grid, Cell Leader, Data aggregation, Tree Routing, EGT.

\section{INTRODUCTION}

Wireless Sensor Network is one of the most important technologies of the twenty first century [1]. It comprises of a set of sensor nodes and sink nodes and has significant applications in weather forecasting, military target tracking, medical monitoring and environmental detection [2][3][4]. It consists of anywhere from a few hundreds to thousands of sensor nodes. However, these nodes have limited computation capability, energy and memory resource [5]. Since the sensor nodes are normally battery powered, it is quite challenging to recharge or replace the batteries.

In Wireless Sensor Network, data transmission takes place in multi-hop fashion where each node forwards its data to the neighbor node nearer to the sink. In a densely populated network, nearby sensor nodes sense data that are correlated, thus leading to data redundancy. Data generated is too much for end-user to process; function for combining data into small set of useful information is required. In-network filtering and processing techniques can definitely help to conserve the scarce energy resources [6]. A more practical way of doing this is aggregating data originating from different nodes in the correlated area. Data aggregation is a process of aggregating sensor data using data aggregation approaches [7]. A number of aggregation algorithms and database systems targeting different sensor network scenarios have been proposed $[8,9,10,11,12,13,14$ 
and 15]. These protocols aim at eliminating redundant data transmission and improving the lifetime of energy constrained Wireless Sensor Network.

This paper proposes a hybrid protocol called "Energy efficient Grid and Tree based routing protocol (EGT)". EGT divides the network into virtual grids. Each grid has a cell leader and is elected based on the maximum residual energy. The sensor nodes in each grid communicate the data to its cell leader. The cell leader aggregates the data sent by its members and the aggregated data is communicated to the sink using Hop Tree. Since this protocol uses both grid and tree approach, it eliminates transfer of redundant data, thereby minimizing the energy consumption and maximizing the lifetime of the network. The rest of the paper is organized as follows: In the next section, we will introduce related work; Section 3 discusses the proposed algorithm. Section 4 presents simulation results and Section 5 presents the conclusions.

\section{RELATED WORK}

"Low Energy Adaptive Clustering Hierarchy (LEACH)" [16] is one of the most popular clustering protocols. It forms clusters by using a distributed algorithm. Each node has the same probability of becoming a cluster-head and the task of being a cluster-head is rotated between nodes according to a round time. This ensures fair energy dissipation between nodes. A noncluster head node in every cluster sends its data to its cluster head. The cluster head compresses the data received from member nodes and sends the compressed data to the base station.

In LEACH-C [17], clusters are formed by centralized control algorithm and it produces better clusters by spreading the Cluster Head throughout the network. The steady phase is similar to that of LEACH, but differs in setup phase. In the setup phase, every sensor node sends its energy information to remote Base Station and this information is used to select the cluster heads. The Base Station then broadcasts the ID of cluster head to other member nodes. In this method, the nodes with more energy have more chances of becoming the cluster head in the current round. But in this phase, every sensor node needs to send its ID and energy information to remote Base Station to compete for the role of cluster heads which consumes energy in long distance transition.

Lindsey et al. proposed "Power-Efficient GAthering in Sensor Information Systems (PEGASIS)" [18], an energy efficient protocol which provides improvement over LEACH. In PEGASIS, each node communicates only with a nearby neighbour in order to exchange data. It takes turns in order to transmit the information to the base station, thus reducing the amount of energy spent per round. The nodes are organized in such a way so as to form a chain, which can either be formed by the sensor nodes themselves using a greedy algorithm starting from a certain node or the Base Station can compute this chain and broadcast it to all the sensor nodes.

Vaibhav V. Deshpande et al. proposed "Energy Efficient Clustering in Wireless Sensor Network using Cluster of Cluster Heads" [19]. To balance energy consumption among the cluster heads, this protocol proposes to have cluster of cluster heads within the cluster of sensor nodes. Given a moment, one cluster head act as master of the given cluster and the master-ship is rotated among cluster heads after specified number of rounds of communication. This improves the energy utilization of sensor network, maximizes the network lifetime and makes the Wireless Sensor Network fault tolerant to some extent.

Neng-Chung Wang [20] proposed "Grid-Based Data Aggregation for Wireless Sensor Networks" (GBDAS) in which the network is divided into 2-D logical grid of cells. In each cell, the node with most residual energy is elected as cell head. The cell head from each cell aggregates its own data with the data sent by all other nodes. All the cell heads are linked to form a chain. The chain leader is designated based on the most residual energy of all the cell heads. In this protocol, 
since the cell heads and the chain leader are designated based on the energy level, the energy depletion of the nodes is distributed evenly.

“An Adaptive Energy aware Data aggregation Tree"(AEDT) [21] uses the maximum energy available node as the data aggregator. The tree incorporates sleep and awake technology, where the communicating node and the parent node are in awake state and the remaining nodes go to sleep state. When the traffic load crosses the threshold value, the packets are accepted adaptively according to the communication capacity of the parent node. It maintains a memory table which stores the value of each selected path. Path selection is based on shortest path algorithm where the node with highest available energy is always selected as the forwarding node.

"A Cluster-based and Tree-based Power Efficient Data Collection and Aggregation Protocol for Wireless Sensor Networks" (CTPEDCA) [22] is based on clustering and Minimum Spanning Tree routing strategy for cluster heads. The performance of CTPEDCA is better than that of LEACH protocol. It prolongs the lifetime of the network and its time complexity is small i.e., $\mathrm{O}(\mathrm{E} \log \mathrm{V})$, where $\mathrm{V}$ is the set of cluster heads.

\section{Proposed Algorithm}

The proposed system i.e. "Energy efficient Grid and Tree based routing protocol" (EGT) consists of two phases: Setup Phase and Steady Phase. Setup Phase forms a grid of sensor nodes and selects a Cell Leader. In each grid, the node with the highest residual energy is elected as the Cell Leader. In the Steady Phase, the sink node constructs a hop tree algorithm. The sensor nodes in each of the grid transmit data to the elected cell leaders. These elected cell leaders aggregate the data and the same is transmitted to the sink using the constructed Hop Tree.

\subsection{Cell Leader Election}

In the Setup Phase, each node broadcasts its residual energy within the radio range r. Every node after receiving the message from all its neighbours, updates the neighbourhood table. The node with the highest residual energy is elected as the cell leader. In addition, each node is randomly assigned a cost value. When more than one node has the same highest residual energy, then the node with more cost will be the Cell Leader. Table 1 shows the Cell Leader Election Algorithm.

Table 1. Cell Leader Election

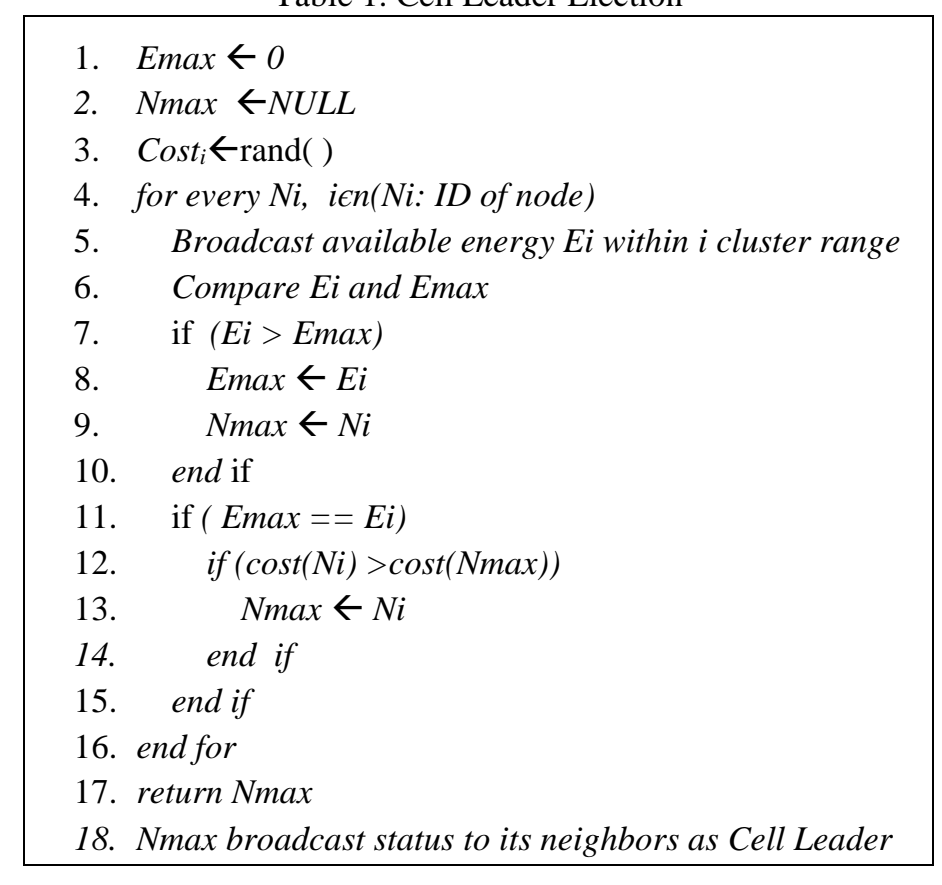




\subsection{Routing Tree Generation}

Once the cell leader is elected and the nodes in the cell want to transfer data to the sink, it becomes essential for the cell leader to communicate the data to the sink using shortest path. A Hop Tree is constructed for finding the shortest path from the cell leaders to the sink. Table 2 below depicts the Hop Tree Construction Algorithm.

Table 2. Hop Tree Construction Algortihm [23]

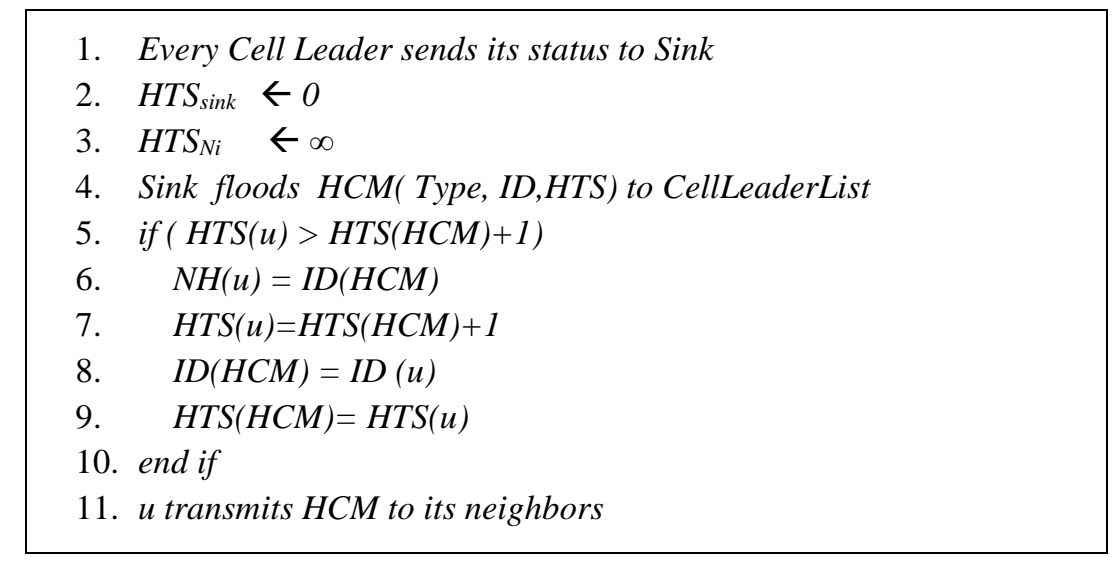

The distance from the sink to each node is computed in hops. The sink floods the Hop Configuration Message (HCM) to all the cell leaders. The HCM message contains three fields: Type, ID and HopToSink where Type is type of the message i.e., HCM, ID is node identifier that started or retransmitted the HCM message and HopToSink is the distance in hops by which an HCM message has passed.

The HopToSink value starts with value zero at the sink and forwards it to its neighbors (at the beginning, all Cell Leaders set the HopToSink as infinity). Each cell leader upon receiving the HCM message verifies if the HopToSink value in the HCM message is less than its own. If so, the node updates its NextHop value with the value of the ID field in HCM message. In addition, the values of HopToSink variable and ID field of HCM message is updated as in Table 2. Cell leader now relays the updated HCM message. Otherwise, the cell leader discards the received HCM message. The above steps are repeated until the whole network is configured. All the updated information i.e. NextHop and HopToSink for every Cell Leader is stored in routing table rTable.

\subsection{Intra-Cluster Communication}

In their allotted time slot, the non-leader nodes in each grid communicate the sensed data to their respective cell leader. Once all the cell members in the network finish transmitting their sensed data, inter-cluster communication phase begins. In a densely populated sensor network, the area detected by sensors may overlap and thus the data sensed may be correlated. This causes data redundancy at the cell leader during intra-cluster communication. Moreover, much energy is consumed in processing similar data and results in more energy consumption. Hence, data aggregation is performed during intra-cluster communication.

Table 3 shows the Intra-Cluster Communication Algorithm. The nodes in each grid transmit the sensed temperature data to the elected Cell Leader. The Cell Leader in each grid aggregates its own data with the data received from all other nodes in that cell using an average function. 
Table 3. Intra-Cluster Communication Algorithm

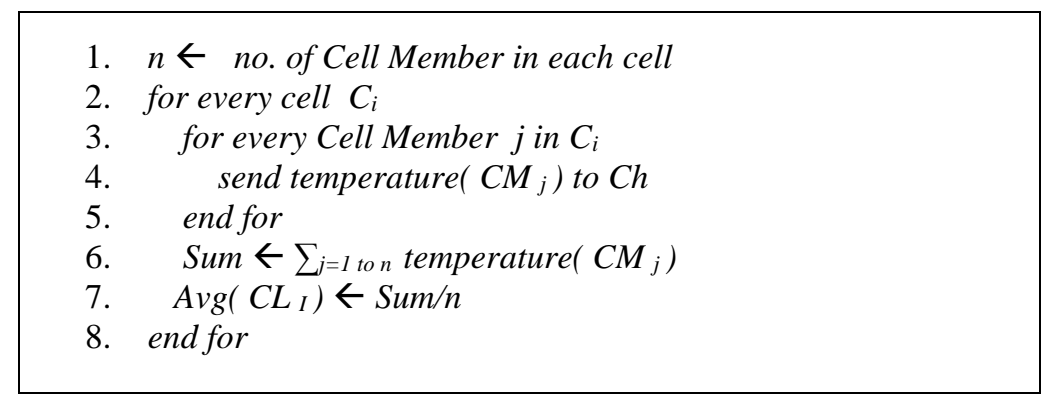

Figure 1 below depicts Intra-Cluster Communication. Each node is labeled with node id followed by its residual energy level. Taking the example of bottom rightmost grid, it can be observed that the node with id 42 has the highest residual energy of 95 Joules and hence it is elected as cluster head. All the other nodes in that grid send its temperature data to the node with id 42 . This node aggregates its data with the data of all other nodes using average function.

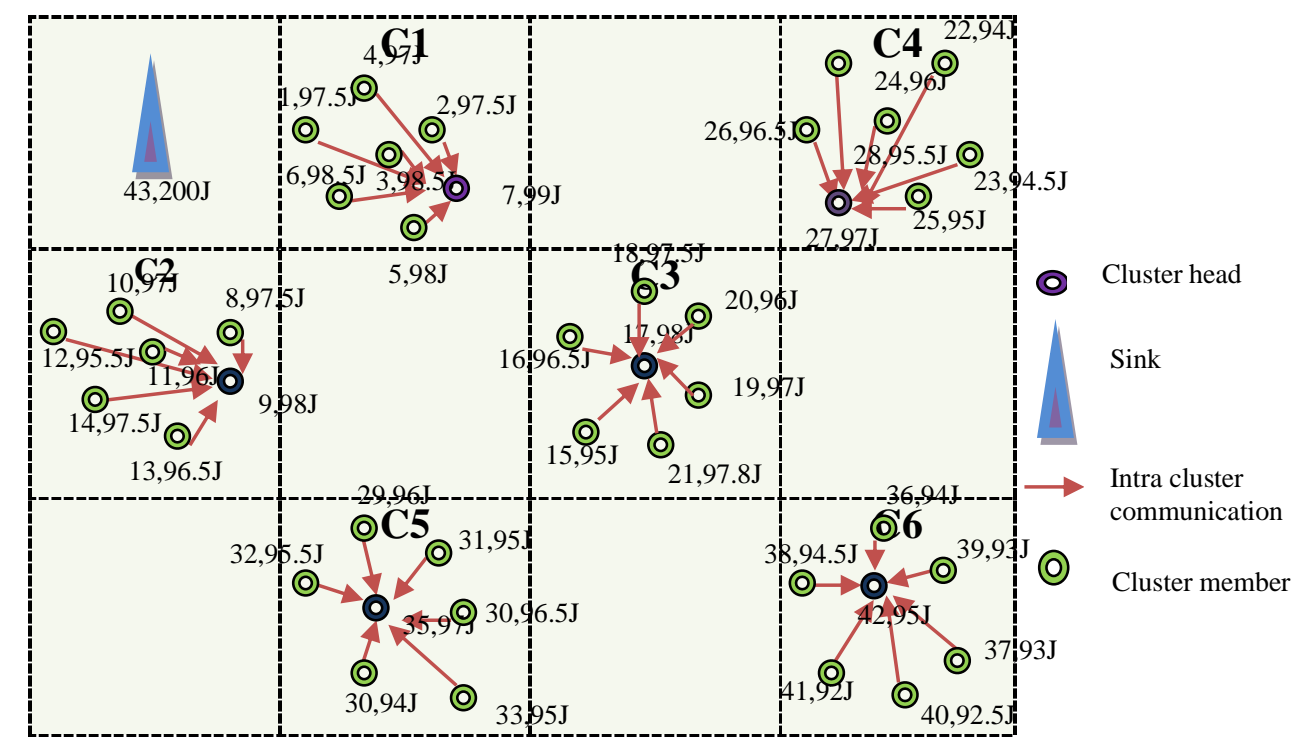

Figure 1. Intra-cluster Communication

\subsection{Inter-cluster Communication}

In Inter-Cluster Communication, routing take place between cell leaders of different cells and the data is relayed to the sink. Paths are established from every cell leader to sink using Hop Tree Construction(HTC) Algorithm of Table 2 and the next hop information for every cell leader is updated in the routing table. Every cell leader accesses the next hop information and forwards its data until its HopToSink (HTS) count decreases to zero. Table 4 below depicts the Inter-Cluster Communication Algorithm.

Table 4. Inter-cluster Communication Algorithm

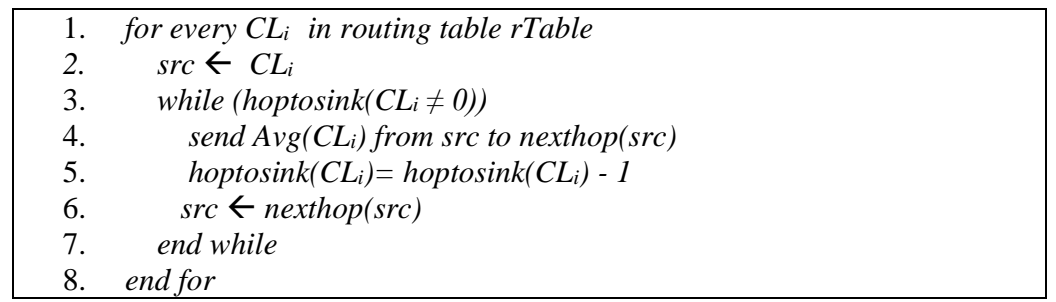


Figure 2 below depicts how the data aggregated from each of the Cell Leader is sent to the sink using the constructed Hop Tree. The aggregated data from Cell Leader C4 and C6 is sent to Cell Leader C3. The Cell Leader C3 aggregates its own data with that of $\mathrm{C} 4$ and $\mathrm{C} 6$ and sends it to Cell Leader $\mathrm{C} 1$. The Cell Leader $\mathrm{C} 1$ aggregates its own data with that sent by $\mathrm{C} 3$ and the same is dispatched to the sink.

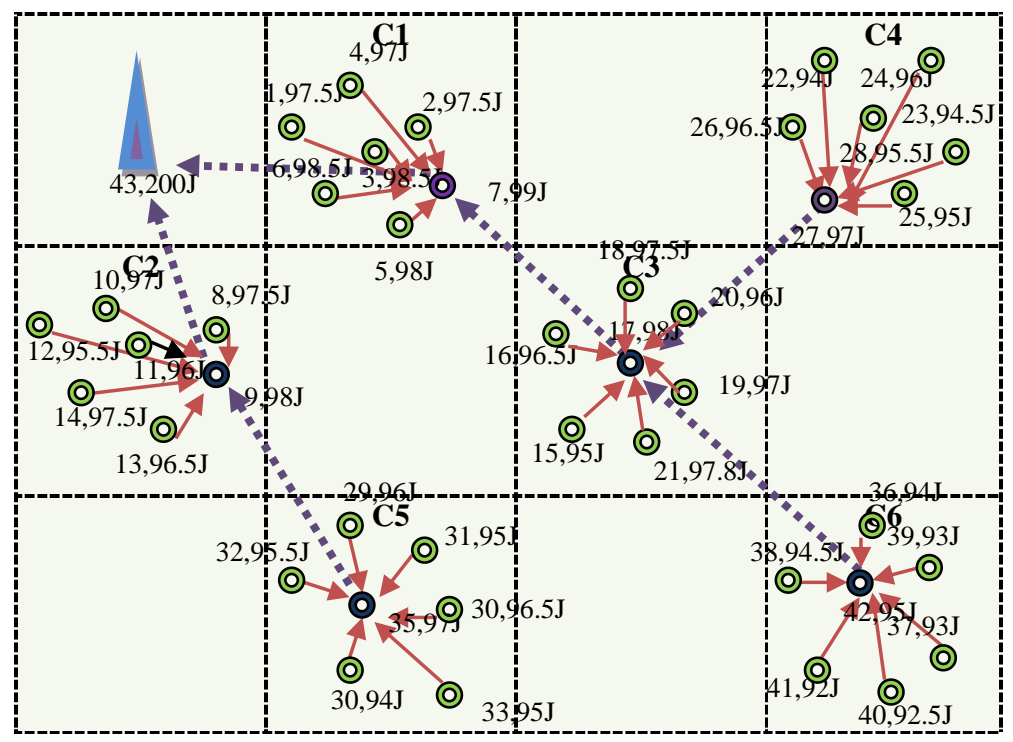

Figure 2. Inter-cluster communication using Hop Tree constructed

\section{Simulation Results}

The simulation environment models a grid based network in which 50 nodes are deployed randomly or uniformly over an area of $1500 \times 1500 \mathrm{~m}^{2}$. The simulation is carried out using NS2. NS2 is an open source event driven simulator tool. Table 5 below shows the list of parameters used for simulation.

Table 5. Simulation Settings

\begin{tabular}{|l|l|}
\hline \multicolumn{1}{|c|}{$\begin{array}{c}\text { Simulation } \\
\text { Parameters }\end{array}$} & \multicolumn{1}{c|}{ Values } \\
\hline Topology size & $1500 \times 1500 \mathrm{~m}^{2}$ \\
\hline Number of sensors & 50 \\
\hline Deployment type & Random / uniform \\
\hline Transmission range & $500 \mathrm{~m}$ \\
\hline Data Packet Size & 1000 bytes \\
\hline Traffic Type & Constant Bit Rate \\
\hline MAC protocol & 802.15 .4 \\
\hline Initial energy & $100 \mathrm{~J}$ \\
\hline
\end{tabular}


Simulation results show the comparison of EGT with LEACH protocol. Figure 3 shows the performance of throughput varying with simulation time. The graph clearly shows that EGT has a greater throughput compared to LEACH. LEACH assumes direct communication between cluster head and sink. Data destined from distant cluster heads gets dropped and hence throughput is less. Whereas in EGT, the cell leaders use multi-hop transmission to relay data to the sink and this results in higher throughput.

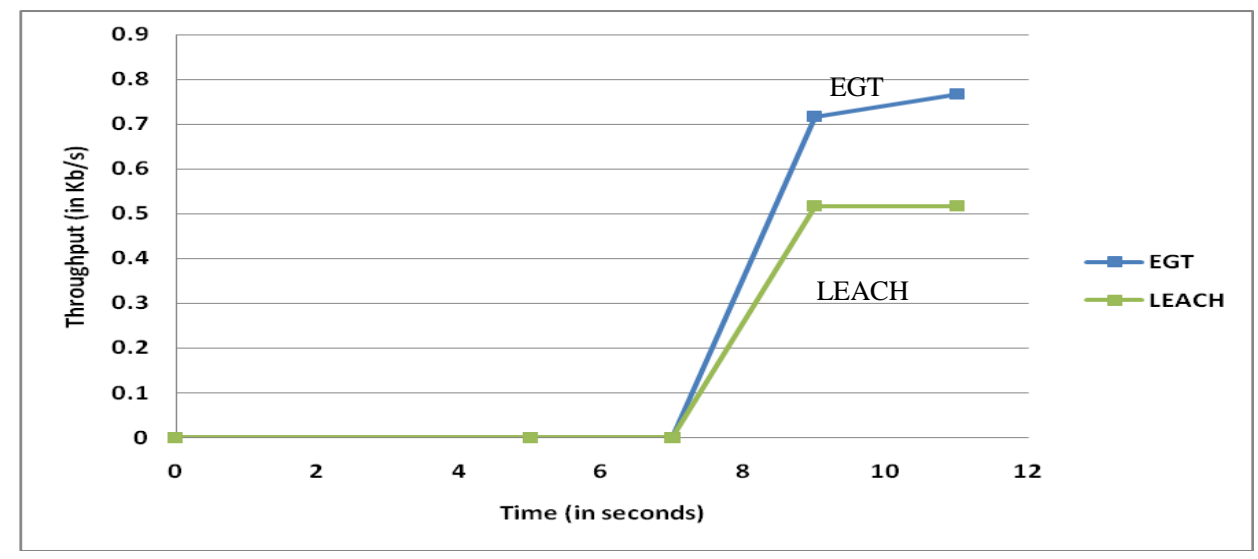

Figure 3. Throughput comparison

Figure 4 shows the performance of EGT compared to LEACH based on packet delivery ratio. At time nine seconds, the cluster heads aggregate the data from the respective grid and transmit to the sink. EGT uses hop tree for inter-cluster communication and hence the aggregated data transmitted by every cluster head will be successfully received by the sink. Therefore, it can be observed from the graph that the packet delivery ratio is 1 for EGT, meaning that the number of packets generated in the network is successfully received at the destination. On the other hand, LEACH assumes direct communication between every cluster head and the sink. As a result, packets transmitted by cluster heads which is out of the coverage area of the sink will be dropped and hence its packet delivery ratio is less than 1 . Therefore, the proposed protocol has a greater packet delivery ratio.

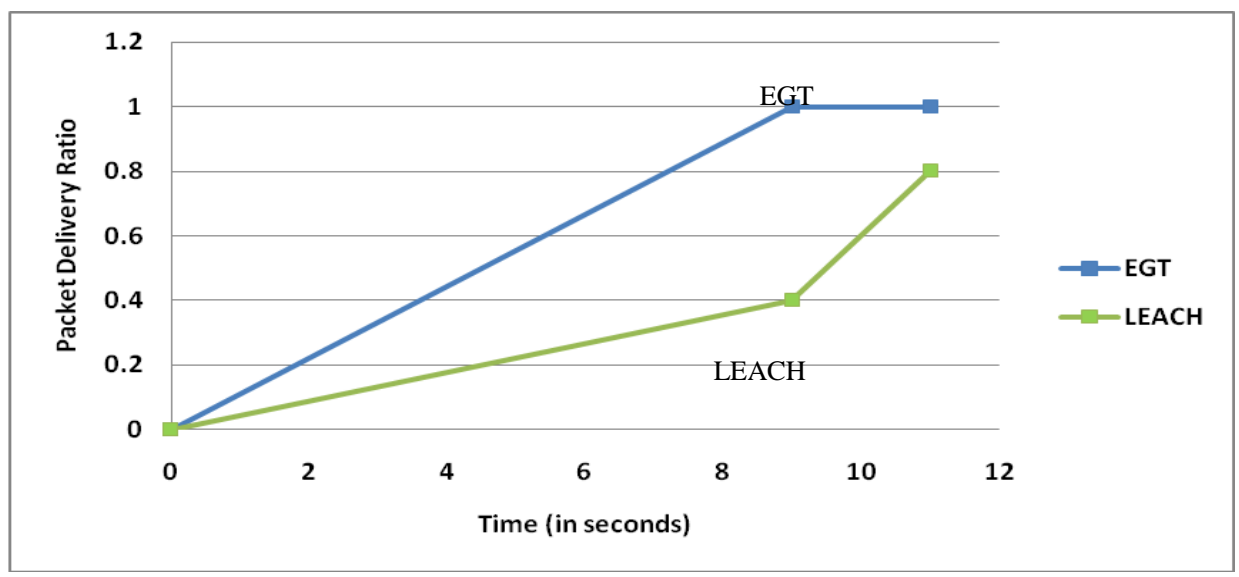

Figure 4. Delivery Ratio Comparison 
Figure 5 shows the average energy remaining in the network after data transmission. In LEACH, since the cluster heads are elected using probabilistic approach, there is a possibility that nodes with lesser remaining energy may be chosen and may die first. As a result, data may have to be retransmitted resulting in more energy consumption. However, such a situation does not arise with EGT and hence EGT has more average energy remaining in the network, after the data is transmitted to the sink.

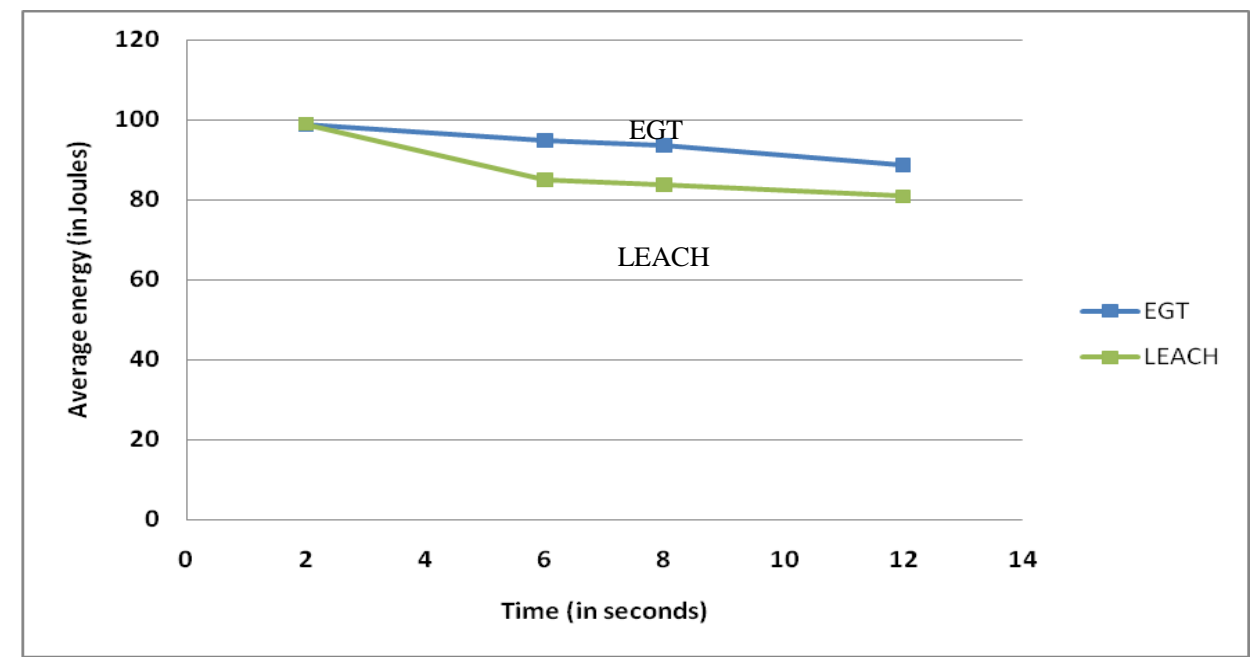

Figure 5. Average Energy Comparison

\section{Conclusion}

In a Wireless Sensor Network, the sensor nodes are battery powered and this act as a major constraint on the energy. Energy is consumed in sensing and transmission. In a densely populated network, nearby nodes sense the same information and this results in transmission of redundant data to the sink. To conserve energy, it is essential to employ energy efficient routing techniques and to avoid transmitting redundant data to the sink. This paper proposes an energy efficient hybrid protocol named "Energy efficient Grid and Tree based routing protocol" (EGT).

EGT divides the sensing area into grids. The cell leader from each grid receives the sensed data, aggregates it and transmits it to the sink using the constructed hop tree. Simulation results show that the proposed protocol is more energy efficient compared to LEACH protocol.

\section{REFERENCES}

[1] "21 ideas for the 21st Century”, Business Week, August 30 1999, pp 78-167.

[2] I.F. Akyildiz and M.C. Vuran, Wireless Sensor Networks, Advanced Texts In Communications And Networking, p.480, 2010.

[3] D. Pompili,T Melodia and I.F. Akyildiz, Three-dimensional and two-dimensional deployment analysis for underwater acoustic sensor networks, Ad Hoc Networks, 7(4):778-790,2009.

[4] L. Li and T. Kunz, Cooperative Node Localization for Tactical Wireless Sensor Networks, Proceedings of IEEE MILCOM'07, 2007.

[5] I.Gupta, D.Riordan and S.Sampalli, “Cluster-head Election using Fuzzy Logic for Wireless Sensor Networks",Communication Networks and Services Research Conference, pp.255-260, May 2005.

[6] Manju Bala and Lalit Awasthi,"Proficient D-HEED Protocol for Maximizing the Lifetime of WSN and Comparative Performance Investigations with Various Deployment Strategies", International Journal of Advanced Science and TechnologyVol.45,August, 2012. 
[7] Nandini. S. Patil and Prof. P. R. Patil, "Data Aggregation in Wireless Sensor Network", IEEE International Conference on Computational Intelligence and Computing Research,Coimbatore, Dec 2010.

[8] S. Madden,R. Szewczyk,M. FranklinandD. Culler, "Supporting aggregate queries overad-hoc sensornetworks", In Workshopon Mobile Computingand Systems Applications (WMCSA),Callicoon, NY, June 2002, Pages: 49 - 58.

[9] Y. Yao and J. Gehrke, "Query Processing in Sensor Networks", In Proceedings of the First Biennial Conference on Innovative Data Systems Research (CIDR 2003),Asilomar,California,January2003.

[10] Demers, J. Gehrke, R. Rajaraman, N. Trigoni, and Y. Yao, "Energy-Efficient Data Management for Sensor Networks: A Work-In-Progress Report", 2nd IEEE Upstate New York Workshop on Sensor Networks. Syracuse, NY, October 2003.

[11] S. Madden, M. Franklin, J. Hellerstein, W. Hong, "The Design of an Acquisitional Query Processor for Sensor Networks", ACM SIGMOD Conference, San Diego, CA, June 2003, Pages: 491 - 502.

[12] Y. YaoandJ. Gehrke, "The Cougar Approach to In-Network Query Processingin Sensor Networks",Sigmod Record, Volume 31, Number 3, September 2002, Pages: 9 - 18.

[13] J. Heidemann,F. Silva,C. Intanagonwiwat,R. Govindan,D. EstrinandD. Ganesan,"Building efficient wireless sensor networks with low-level naming", In Proceedings of the eighteenth ACM symposiumon Operating systems principles,Banff, Alberta,Canada, October 2001, Pages: 146-159.

[14] C. Intanagonwiwat, D. Estrin, R. Govindan, and J. Heidemann, "Impact of network density on data aggregation in wireless sensor networks", in ICDCS-22, November 2001.

[15] C. Intanagonwiwat, R. Govindan andD. Estrin, "Directed diffusion:A scalableand robust communication paradigm for sensor networks", In Proceedings of the Sixth Annual International Conferenceon Mobile Computing and Networks (MobiCOM 2000),Boston,MA,August 2000, pages: 56-67.

[16] W R Heinzelman, A Chandrakasan and H. Balakrishnan, "Energy-efficient communication protocol For wireless microsensor networks,"in Proceedings of the33rdAnnualHawaii International Conference on System Sciences (HICSS '00), January 2000.

[17] W. B. Heinzelman, A. P. Chandrakasanand H. Balakrishnan, "An application-specific protocol architecture for wireless microsensor networks,"IEEE Transactions on Wireless Communications, vol. 1, no. 4, pp. 660-670, 2002.

[18] Lindsey S and Raghavendra C., "PEGASIS: Power-Efficient GAthering in Sensor information Systems", In Proceedings of the IEEE Aerospace Conference, Los Angeles, MT, USA, 2002; pp. 1125-1130.

[19] Vaibhav V. Deshpande,Arvind R. Bhagat Patil,"Energy Efficient Clusteringin Wireless Sensor Network using Cluster of Cluster Heads", 10thInternationalConference on Wireless and Optical Communications Networks (WOCN), 26-28th July 2013, Bhopal, pp 1-5.

[20] Neng-Chung Wang et. al, "Grid-Based Data Aggregation for Wireless Sensor Networks" (GBDAS), Journal of Advances in Computer Networks, Vol 1, No. 4, December 2013.

[21] Deepali Virmani,TanuSharma,"An Adaptive Energy aware Data aggregation tree” (AEDT),Science and Development Network (SciDev.Net), Jan 2013.

[22] Wei Wang, Bingwen Wang Zuo Liu, Lejiang Guo and Wei Xiong, "A Cluster-based and Tree- based Power Efficient Data Collection and Aggregation Protocol for Wireless Sensor Networks",Information Technology Journal, Jan 2011; 10: 557-564.

[23] Yalin Nie, Sanyang Liu, Zhibin Chen and Xiaogang Qi, "A Dynamic Routing Algorithm for DataAggregation Optimization in event-Driven Wireless Sensor Networks", Journal of Communications Vol. 8, No. 8, August 2013.

\section{AUTHORS}

Reshma J received B.E and M Tech degree in Computer Science and Engineering from Bangalore University and Visvesvaraya Technology University respectively. She has more than ten years of teaching experience. Her research interests include wireless networks and sensor networks. 
International Journal of Wireless \& Mobile Networks (IJWMN) Vol. 7, No. 3, June 2015

Dr.Kavitha C received her B.E. and M.E degree in Computer Science \& Engineering from Bangalore University, Karnataka India and her Ph.D from Visvesvaraya Technological University, Belgaum, Karnataka - India in 2010. She has more than eighteen years of teaching experience. Her research interests include wireless networks, sensor networks.

Malashri S received B.E degree in Information Science and Engineering from Visvesvaraya Technology University in 2011 and pursuing M. Tech degree in Computer Network Engineering in Visvesvaraya Technology University. She has two years of teaching experience. Her research interests include Wireless networks and sensor networks.
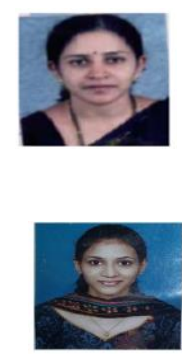